\title{
Microscopic dynamics of glycerol in its crystalline and glassy states
}

\author{
F. J. Bermejo \\ Instituto de Estructura de la Materia, Consejo Superior de Investigaciones Científicas, Serrano 123, E-28006 Madrid, Spain \\ A. Criado \\ Instituto de Ciencia de Materiales, Consejo Superior de Investigaciones Científicas, \\ and Departamento Física de la Materia Condensada, Universidad de Sevilla, Box 1065, E-41080 Sevilla, Spain
}

A. de Andres

Instituto de Ciencia de Materiales, Consejo Superior de Investigaciones Científicas, Campus de Cantoblanco, Universidad Autónoma de Madrid, E-28049 Madrid, Spain

E. Enciso

Departamento de Química Física I, Universidad Complutense, Ciudad Universitaria, E-28040 Madrid, Spain

\author{
H. Schober* \\ Centre d'Etudes Nucleaires Grenoble, DRFMC, SPSMS, MDN, 85X, F-38041 Grenoble Cedex, France
}

(Received 15 September 1995)

\begin{abstract}
The dynamics of crystalline glycerol are studied by means of Raman spectroscopy and lattice dynamics calculations employing a semiflexible model to represent the low-lying molecular vibrations. The latter is validated against structural, thermodynamic, and spectroscopic data. The results serve to set an absolute frequency scale for glassy glycerol, which is also studied by Raman and incoherent inelastic-neutron scattering. Some implications of the present findings regarding ensuing discussions on glassy dynamics are finally commented on.
\end{abstract}

\section{INTRODUCTION}

The understanding of the dynamical processes in highly viscous liquids such as glycerol as well as the glassy state achieved upon crossing from above the glass transition $\left[T_{g} \approx 185 \mathrm{~K}\right.$ at saturated vapor pressure (SVP)] has been the focus of a great deal of attention for many decades. Its large viscosity at temperatures about melting $(\approx 3 \mathrm{~Pa} \mathrm{~s}$ at room temperature and zero pressure ${ }^{1}$ ) makes nucleation processes leading to crystallization extremely rare, thus enhancing the stability of the strongly supercooled liquid, which can then be studied for very long times.

Despite the wealth of thermodynamic studies involving static $^{2}$ and dynamic ${ }^{3}$ measurements, or other investigations employing optical ${ }^{4-6}$ or dielectric relaxation means, ${ }^{7}$ and up to the authors knowledge, no attempt to relate such findings to the underlying microscopic motions has yet appeared. However, consideration of heat capacity data ${ }^{2}$ for the material in glass and crystal states at temperatures somewhat below $T_{g}$ (i.e., $170 \mathrm{~K}$ ), gives $C_{p}^{\text {glass }} \approx 91.4 \mathrm{~J} \mathrm{~mol}^{-1} \mathrm{~K}^{-1}$, $C_{p}^{\text {cryst }} \approx 83.1 \mathrm{~J} \mathrm{~mol}^{-1} \mathrm{~K}^{-1}$, something which indicates that (a) a substantial contribution to this thermodynamic magnitude involves motions additional to rigid-body molecular rotations and translations, which would yield a hightemperature limit of $\approx 49.9 \mathrm{~J} \mathrm{~mol}^{-1} \mathrm{~K}^{-1}$, and (b) the heat capacity excess of the glass with respect to crystal only amounts to some nine percent of $C_{p}$, strongly suggesting that most of the atomic dynamics in the disordered state can be understood in terms of vibrations.
On the other hand, such low-frequency vibrational character persists well into the supercooled and even normal liquid phases as evidenced by the presence of well resolved peaks at low frequencies ("Boson" peaks) in the neutron and Raman spectra up to temperatures as high as $1.5 T_{g} \cdot{ }^{4,5,8} \mathrm{As}$ a matter of fact, as shown in Ref. 8, the spectra for frequencies above $1 \mathrm{THz}$ can easily be superimposed after scaling by the Bose factor for temperatures within the range 170-313 $\mathrm{K}$, thus indicating that at least for such frequencies, the vibrations in the glass and liquid share rather similar characteristics.

Under such circumstances, establishing an absolute frequency scale as given by the crystal, to which the characteristic anomalies of the glassy state can be referred to seems a prerequisite for any quantitative discussion on the dynamics, at least for frequencies above a few hundreds of GHz. This would then serve to overcome the difficulties derived from the use of oversimple models as a reference (i.e., Debye-like frequency distributions), the validity of which are confined to rather low temperatures. Such an exercise would also seem to be essential if some dynamical property such as the generalized susceptibility (or dynamic structure factor) is to be discussed in terms of results able to describe the relevant motions of monoatomic systems only, ${ }^{8}$ such as those pertaining to mode-coupling-theory (MCT) approaches.

Our emphasis will thus be centered on the development of a microscopic model able to give due account of the crystal structure and dynamics which may be adapted for computer simulation studies of the glass (and cold liquid) at temperatures about $T_{g}$. To achieve that, the crystal structure, ther- 
modynamics, and Raman spectra are studied as benchmarks for the development of such a force field. The frequency distributions calculated for such a lattice-dynamical (LD) model are then considered as those characteristic of the "ground state" to which those measured for the glass can be referred to.

Finally, some implications of the present results regarding current discussions on the dynamics of glasses near the glass transition are considered.

\section{EXPERIMENTAL AND CALCULATIONAL DETAILS}

\section{A. Experiments}

Several attempts to grow stable crystals, all of them involving solution of glycerol into a low-viscosity solvent, were performed. After several trials with different solvents, the procedure sketched in Ref. 9 was followed since it gives rise to crystallization under reproducible conditions. Seed crystals were grown by dissolving under an inert atmosphere equal amounts of glycerol (25 c.c.) and ammonia on a liquid nitrogen bath for about fifteen hours. The mixture was then brought to about $221 \mathrm{~K}$ by transferring the vessel into a container filled with a mixture of hexanol and dry ice. The seeds then started to grow, and full crystallization was achieved in about five hours. The solvent was then removed by bringing the vessel into contact with another reservoir kept under liquid nitrogen, repeating this operation several times until completion.

The samples were purified in order to remove the traces of occluded ammonia by grinding the crystals under a dry nitrogen atmosphere and subsequent pumping under vacuum at $273 \mathrm{~K}$ for $24 \mathrm{~h}$. The Raman measurements were carried using a $X-Y$ Dilor multichannel spectrometer employing the $514.5 \mathrm{~nm}$ and $488 \mathrm{~nm}$ emissions of a coherent $\mathrm{Ar}^{+}$laser keeping the incident power below $80 \mathrm{~mW}$. The spectra were recorded for temperatures within the $8 \mathrm{~K} \leqslant T \leqslant 300 \mathrm{~K}$ interval using a backscattering geometry for the crystal and the rightangle one for the glass and liquid. Temperature control was achieved by means of an Oxford Instruments continuum flux cryostat, and the optical slits were set to achieve a nominal resolution of $0.08 \mathrm{THz}$. The measured Raman intensities were normalized by the $[n(\omega)+1]$ temperature (Bose) factors for the Stokes side of the spectrum so that a normalized spectrum is given by $I_{\text {norm }}(\omega)=I(\omega) /[n(\omega)+1]$.

Inelastic incoherent neutron scattering (IINS) measurements have been carried out in order to contrast the frequency distributions derived from Raman scattering. Although some neutron data have recently been reported, ${ }^{8}$ both the relatively high temperatures explored (above $170 \mathrm{~K}$ ) in those measurements and the restricted kinematic range (the employed incident energy was $\approx 1.13 \mathrm{meV}$ ) make them of scarce utility for the present purposes. A set of measurements on a fully hydrogenated sample of glycerol for a range of temperatures comparable to that of the Raman data was carried out using the DN6 thermal time-of-flight spectrometer located at the Siloé reactor. An incident energy of $18.3 \mathrm{meV}$ was employed and the sample container was a thin aluminum plate, and the data were fully corrected for container scattering, instrumental effects as well as multiple scattering contributions. ${ }^{10}$

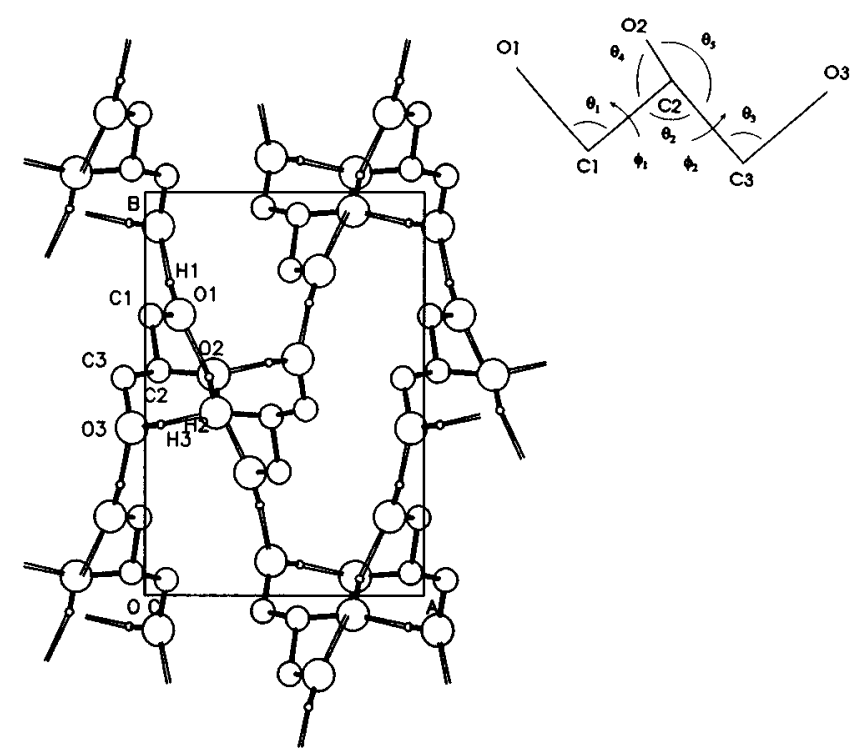

FIG. 1. Optimized crystal structure projected along the $c$ direction. The numbering identifies the atoms of a molecule taken as reference. The bonds drawn with empty lines represent intermolecular hydrogen bonds. The inset shows a schematic diagram of the molecular structure identifying the seven internal molecular coordinates considered in the LD model, which comprises $\theta_{i}$ bond bending and $\phi_{i}$ bond torsions.

\section{B. Computations}

The experimental crystal structure belongs to the orthorhombic $P 22_{1} 2_{1}$ space group $^{11}$ with four $\mathrm{C}_{3} \mathrm{O}_{3} \mathrm{H}_{8}$ units per primitive cell. The molecule adopts an extended conformation, ${ }^{12}$ where the second row atoms $(\mathrm{C}, \mathrm{O})$ are approximately coplanar, showing a shape of a flattened " $W$," which is the most stable conformation for the isolated molecule. ${ }^{11}$ The crystal packing shows infinite hydrogenbonded chains, each hydroxyl hydrogen participating in one hydrogen bond and each oxygen being involved in two hydrogen bonds both as a donor and acceptor, yielding a total of twelve hydrogen bonds per unit cell (see Fig. 1).

We started from the model of Root et al. ${ }^{13}$ which considers the $\mathrm{CH}$ and $\mathrm{CH}_{2}$ groups as point masses. The energyminimized crystal structure departed from the experimental one considerably more than the usual threshold values in crystal packing studies using atom-atom potentials. Such a failure arises from the neglect of interactions of the hydrogen atoms, which are crucial in determining the packing in molecular crystals. A more realistic model, with all atoms as force centers interacting through a potential function, $V(r)=-A / r^{6}+B \exp (-C r)$, was chosen and the parameter sets selected amongst the most reliable ones. ${ }^{14}$ These functions account for all atomic interactions exception made of the hydrogen-bond pairs and those involving intramolecular interactions of atoms bonded to a common one (1-3 interactions).

A Lippincott-Schroeder potential ${ }^{15}$ with parameters taken from Ref. 16 was used for the hydrogen bond interaction, and the electrostatic point-charge model used for the hydroxyl group in the previous work ${ }^{13}$ was also adopted $(q= \pm 0.34 e)$. 
To account for the molecular flexibility two bond-torsion and five bond-angle bendings are allowed for the second row skeleton (see Fig. 1). The former are accounted by functions of type $V_{\text {tors }}=\frac{1}{2} K_{\phi}(1+\cos 3 \phi)$ where $\phi$ refers to the torsion (dihedral) angles defined around each $C-C$ bond. The bending potential is $V_{\text {bend }}=\frac{1}{2} K_{\theta}\left(\theta-\theta_{0}\right)^{2}$, where $\theta$ is the bond angle and $\theta_{0}=109.47^{\circ}$ its equilibrium value are used. The $K_{\phi}, K_{\theta}$ force constants had to be optimized to give an adequate account of the experimental crystal structure, and the optimal parameter set after minimization using the WMIN code (Ref. 17) was $K_{\phi}=0.54 \mathrm{~kJ} \mathrm{~mol}^{-1}$ and $K_{\theta}=356.9$ $\mathrm{kJ} \mathrm{mol}^{-1} \mathrm{rad}^{-2}$, the latter accounting for $C-C-C$ and $C-C-O$ bending terms. The energy-minimized crystal structure is now in good agreement with experiment and the optimal $a, b$, and $c$ unit cell parameters differ $0.4,1.2$, and $0.1 \%$ from the experimental values. The maximum rigidbody translation and rotation shifts were $0.9 \AA$ and $5.5^{\circ}$ and the variations of the torsion angles were $2.6^{\circ}$ and $5^{\circ}$ whereas the maximum variation of the bond angles was $1.1^{\circ}$.

The lattice dynamics (LD) was studied following the molecular Born-von Kármán route, setting up the dynamical matrix in terms of translation and rotational coordinates as well as the internal normal coordinates of the isolated molecule. ${ }^{18}$ The $Z(\omega)$ crystal vibrational density of states (DOS) and the dispersion curves were calculated after performing a dense sampling over all allowed excitations in the Brillouin zone. According to the chosen molecular degrees of freedom, 13 per molecule, 49 modes are expected to appear at $\vec{q}=0$ in the low-frequency range, all of them Raman active giving rise to an intricate pattern in the crystal spectrum.

\section{RESULTS AND DISCUSSION}

\section{A. Crystal dynamics}

Some of the crystal spectra for frequencies below some $25 \mathrm{THz}$ are shown in Fig. 2. Raman lines above this frequency arise from higher-frequency internal modes, which are not analyzed here due to their somewhat marginal importance for the present purposes. Well defined crystal excitations are observed at the lowest explored temperature, some of which survive as strong peaks up to temperatures close to melting. Such a result contrasts with that recently analyzed regarding a van der Waals glass (ortho-terphenyl), ${ }^{19}$ where strong phonon lifetime effects start to be important at temperatures as low as $12 \mathrm{~K}$. In particular, it is remarkable how low frequency modes seen at $\approx 1.65 \mathrm{THz}, \approx 3.28 \mathrm{THz}$, and $\approx 4.37 \mathrm{THz}$ do not show strong reductions in lifetime at temperatures as high as $295 \mathrm{~K}$ alongside that seen for other modes seen as clear peaks at $8 \mathrm{~K}$.

To enable a comparison between calculated and experimental Raman frequencies, a value for the achieved instrumental resolution of $\approx 0.135 \mathrm{THz}$ was estimated from the full width at half maximum (FWHM) of well resolved peaks, and the result is shown in Table I. There, calculated modes separated by a frequency smaller than the experimental resolution have been grouped together and assigned to a single experimental frequency. Some of the calculated modes do not have an experimental counterpart, probably because of their weak intensities. As can be judged from the table, the agreement between experiment and calculation can be con-

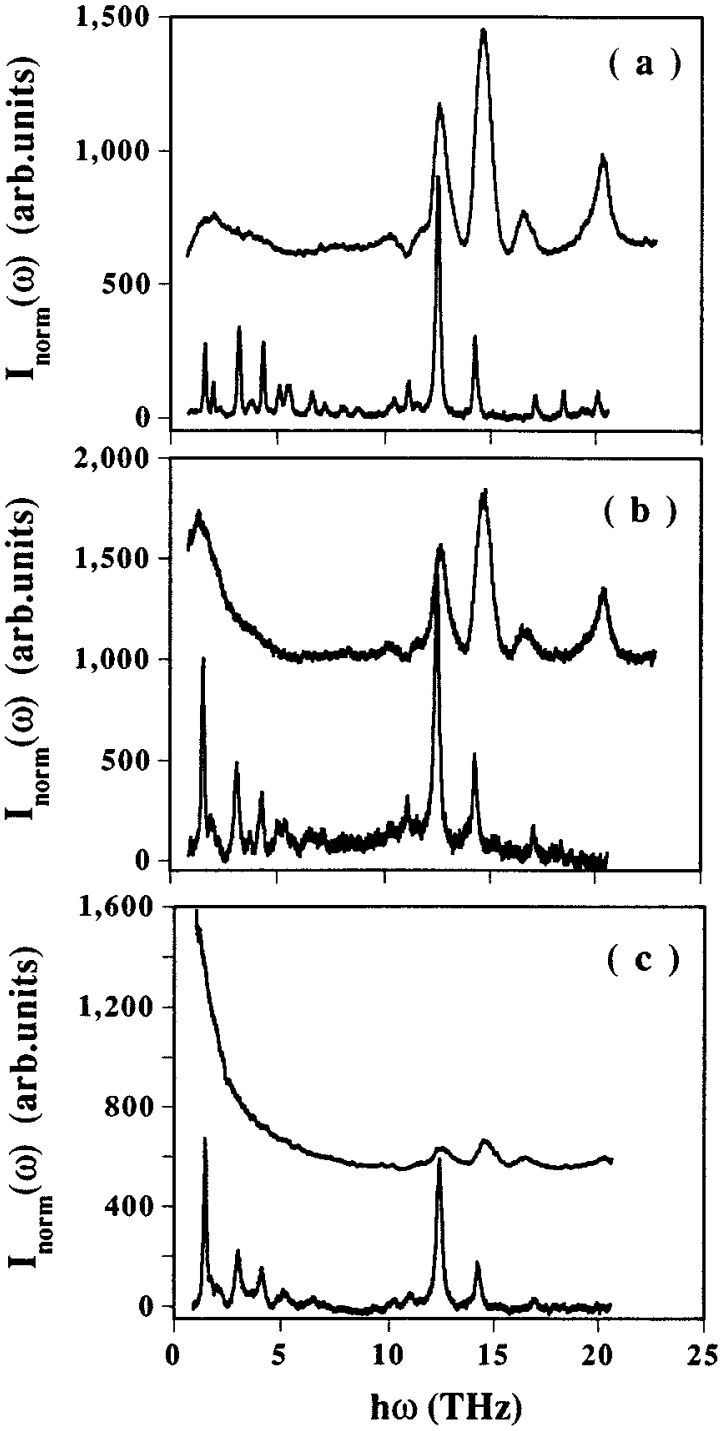

FIG. 2. Raman intensities after correction by the Bose factor $I_{\text {norm }}(\omega)$ for the polycrystal (down) and glass or liquid spectra (up), for a set of temperatures comprising the low-temperature crystal and glass. The glass spectra are shifted by 600 units to enable comparison. (a) Crystal and glass spectra for $T=8 \mathrm{~K}$. (b) Spectra for the hot crystal and supercooled liquid, both at $T=190 \mathrm{~K}$. (c) For the normal liquid (room temperature) and the crystal close to melting $T=295 \mathrm{~K}$.

sidered as rather good, thus validating the present LD model as an adequate tool to represent the crystal dynamics.

To quantify the extent of the hybridization of the lattice and internal molecular modes the normalized mode eigenvector components were calculated. These are given in terms of rigid-body translations and rotations $T_{x, y, z}, R_{x, y, z}$ coefficients and those $\theta_{1-5}, \phi_{1,2}$ regarding the internal coordinates defined in Fig. $1 .{ }^{20}$ Consideration of such quantities evidence that even the lowest frequency mode shows noticeable couplings with the internal ones, especially for the bond torsions which have coefficients $\phi_{1}=-0.328, \phi_{2}=0.315$. The presence of large deformational components alongside substantial contributions from translational motions are characteristic of the three lowest frequency modes, which show coefficients corresponding to the largest rigid-body transla- 
TABLE I. Calculated and experimental Raman frequencies expressed in THz. Those modes with a hyphen have not found a counterpart in the experimental spectra and those marked with an asterisk differ less than the experimental resolution $(0.135 \mathrm{THz})$ with respect to the preceding one. Subsequent modes marked with an asterisk would correspond to a single peak in the experimental spectra and therefore only one of them is assigned an experimental value.

\begin{tabular}{|c|c|c|c|c|c|c|c|}
\hline Mode & Symm. & Calc. & Obs. & Mode & Symm. & Calc. & Obs. \\
\hline 1 & $B_{2}$ & 1.64 & 1.65 & 26 & $B_{1}$ & $6.28 *$ & - \\
\hline 2 & $A_{g}$ & 1.86 & - & 27 & $B_{2}$ & $6.30 *$ & 6.42 \\
\hline 3 & $B_{1}$ & 2.15 & 2.04 & 28 & $B_{3}$ & 6.82 & 6.66 \\
\hline 4 & $B_{3}$ & 2.40 & 2.34 & 29 & $B_{2}$ & 7.15 & 7.23 \\
\hline 5 & $B_{2}$ & $2.43^{*}$ & - & 30 & $A_{g}$ & 8.47 & 8.10 \\
\hline 6 & $A_{g}$ & $2.49 *$ & - & 31 & $B_{3}$ & $8.59 *$ & - \\
\hline 7 & $B_{1}$ & 2.91 & - & 32 & $B_{2}$ & $8.60 *$ & - \\
\hline 8 & $B_{3}$ & 3.22 & 3.27 & 33 & $B_{1}$ & 8.77 & 8.82 \\
\hline 9 & $B_{3}$ & 3.46 & - & 34 & $B_{1}$ & 10.33 & 10.26 \\
\hline 10 & $B_{2}$ & $3.55^{*}$ & - & 35 & $B_{3}$ & $10.34^{*}$ & - \\
\hline 11 & $A_{g}$ & $3.66^{*}$ & - & 36 & $A_{g}$ & $10.44^{*}$ & 10.47 \\
\hline 12 & $A_{g}$ & $3.79 *$ & 3.75 & 37 & $B_{2}$ & $10.51 *$ & - \\
\hline 13 & $B_{2}$ & 3.96 & 3.90 & 38 & $B_{1}$ & 10.72 & - \\
\hline 14 & $B_{1}$ & $4.04 *$ & - & 39 & $A_{g}$ & 10.98 & 11.16 \\
\hline 15 & $B_{3}$ & 4.29 & 4.38 & 40 & $B_{2}$ & 11.70 & 11.64 \\
\hline 16 & $A_{g}$ & 4.50 & - & 41 & $B_{3}$ & $11.85^{*}$ & - \\
\hline 17 & $B_{2}$ & $4.53^{*}$ & 4.74 & 42 & $B_{2}$ & 12.13 & - \\
\hline 18 & $B_{1}$ & 5.13 & 5.16 & 43 & $B_{3}$ & 12.29 & - \\
\hline 19 & $B_{3}$ & $5.25 *$ & - & 44 & $A_{g}$ & $12.38 *$ & - \\
\hline 20 & $A_{g}$ & $5.32 *$ & - & 45 & $B_{1}$ & $12.44^{*}$ & 12.54 \\
\hline 21 & $B_{3}$ & 5.61 & 5.55 & 46 & $B_{2}$ & 14.14 & - \\
\hline 22 & $B_{1}$ & $5.62 *$ & - & 47 & $B_{3}$ & $14.15^{*}$ & - \\
\hline 23 & $A_{g}$ & 5.90 & - & 48 & $A_{g}$ & $14.26^{*}$ & 14.25 \\
\hline 24 & $B_{1}$ & $5.98 *$ & - & 49 & $B_{1}$ & $14.40 *$ & - \\
\hline 25 & $A_{g}$ & 6.18 & - & & & & \\
\hline
\end{tabular}

tional component of $T_{y}=-0.770, T_{x}=0.741, T_{z}=0.775$ respectively. Such couplings become very strong at frequencies as low as $2.15 \mathrm{THz}$ as evidenced by a coefficient for bond torsions $\phi_{2}=0.775$ and one for translations of $T_{z}=-0.548$. In short, modes with frequencies below 7.15 THz have a marked torsional $\left(\phi_{i}\right)$ contribution, whereas bending contributions $\left(\theta_{i}\right)$ are confined within 5.62 to 14.4 THz. Also, the strong peaks seen at 12.54 and $14.25 \mathrm{THz}$ arise from predominantly bending modes showing small frequency dispersion which results in intense and narrow peaks in the vibrational density of states.

The calculated mode frequencies for the isolated molecule are 13.3, 10.1, 9.62, 8.24, 6.09, 2.90, and $2.01 \mathrm{THz}$ where the last two modes are predominantly torsional and the rest are mainly bending modes. It is worth recalling that, as illustrated previously for other systems, ${ }^{21}$ the absence of different time scales for the lattice and internal modes leads to important hybridization effects which affect even these relatively high-frequency modes, making the spectrum of internalmode excitations depart substantially from that for a free molecule, since they can hardly be separated from the lattice ones.

As regards the elastic properties of the material, the analysis of the dispersion branches corresponding to the higher symmetry directions shows that sound waves with phase velocities of $\approx 2.36 \times 10^{3} \mathrm{~ms}^{-1}(T)$ and $\approx 3.70 \times 10^{3}$
$\mathrm{ms}^{-1}(L)$ are propagated in the crystal at frequencies comparable or below $1 \mathrm{THz}$, and that purely translational motions are confined to a small region of frequencies and wave vectors. The lowest frequency Raman line is thus seen to sit on a dispersion region where the zone-boundary sound branches merge with the lowest optical excitations.

The frequency gap seen in the Raman spectra between 14.2 and $17.1 \mathrm{THz}$ separates the torsional and bending modes from the nonhydrogen bond stretching modes, the latter located in the high-frequency range of the measured spectra.

The calculated $Z(\omega)$ crystal vibrational density of states which is drawn in Fig. 3 shows an initial Debye behavior extending up to $0.6 \mathrm{THz}$, followed by a sequence of peaks, a gap starting at $7.5 \mathrm{THz}$, and a series of strong peaks mostly arising from the internal molecular modes. From the initial $\propto \omega^{2}$ behavior, a Debye frequency of $\omega_{D}=4.91 \mathrm{THz}$ is calculated which corresponds to a Debye temperature of some $235 \mathrm{~K}$.

As shown in the inset of Fig. 3, the dynamic structure factor evaluated from the computed distribution under the incoherent approximation, (i.e., $\quad S_{\text {inc }}(Q, \omega)$ $\propto Z(\omega)[n(\omega)+1] / \omega)$ shows a strong peak centered at some $1.5 \mathrm{THz}$, the relevance of which in the context of glassy dynamics is discussed below, and shows a substantial spread in frequencies which extends down to the elastic line (not shown in the figure). 


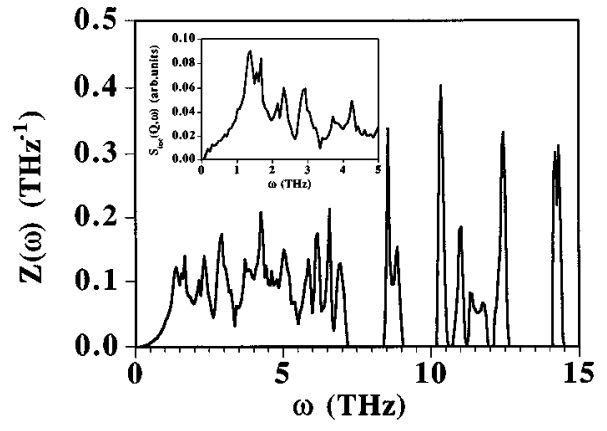

FIG. 3. Vibrational density of states $Z(\omega)$ as calculated for the crystal using the procedures described in previous sections. The inset shows the crystal dynamic structure factor as calculated from the frequency distribution under the incoherent approximation (see text).

\section{B. Glass frequency distributions}

A comparison between crystal and glass spectra shown in Fig. 2 reveals that (a) drastic changes occur at low (below some $6 \mathrm{THz}$ or so) frequencies, the sharp lines arising from Raman active modes with $\vec{q} \simeq 0$ being replaced by a continuous distribution of intensities, and (b) substantial changes above $10 \mathrm{THz}$ are observed as a consequence of vitrification which translate into noticeable changes in frequencies, widths, and intensities of the strong crystal peaks seen at 12.5 THz, $14.26 \mathrm{THz}$, and $17.17 \mathrm{THz}$. This is mostly caused by the presence in the glassy state of molecular isomers of different skeletal conformation, as can be shown by consideration of the molecular internal frequencies calculated for different atomic configurations. In fact, an estimation of the spread in frequencies arising from variations in the $\phi_{1,2}$ angles yielded values for every mode distributed within some $\approx 1.8 \mathrm{THz}$, a result comparable with the observed shifts.

\section{Low-frequency scattering}

The feature located at $\approx 1.2-1.6 \mathrm{THz}$ in the glass spectra shown in Fig. 2 is commonly referred to as the "Boson peak." Its microscopic origin has recently been the focus of a number of research efforts, ${ }^{5,22-25}$ and its absence in the crystal Raman spectra is mostly due to crystal selection rules, since the generalized dynamic structure factor for a powder calculated under the incoherent approximation commented on in previous sections shows that a peak appears in the polycrystal spectrum at some $1.5 \mathrm{THz}$ (see Fig. 3).

As is now well established, ${ }^{26}$ the low-frequency Raman spectrum of an isotropic body such as a glass consists of two contributions which are a broad quasielastic line and the lowfrequency tail of the first-order vibrational spectrum. The former contribution is usually confined to frequencies far smaller than those reachable by the present experiments whereas the latter arises from the breakdown of the crystal selection rules caused by the randomness in the coupling between electric polarization and atomic displacements, as well as from the fundamental fact that only modes with wavelengths larger than some "correlation length" can be rightly represented as plane waves. In consequence, the normalized Raman intensity $I_{\text {norm }}(\omega)$ is related to the density of vibrational states through a relationship such as $I_{\text {norm }}=C(\omega) Z(\omega) / \omega$, where $C(\omega)$ stands for the light-tovibrations coupling, which only in the extreme cases of complete randomness of the atomic couplings (or the atomic amplitudes) can be considered as frequency independent. Therefore, the origin of the low-frequency peak observed experimentally could be ascribed to peaks in the $C(\omega)$ lightto-vibrations coupling, to peaks in the $Z(\omega) / \omega^{2}$ function if $C(\omega)$ were independent of the frequency, or in both. ${ }^{26}$

Our aim here is to verify whether the frequency dependence of $C(\omega)$ is a material property ${ }^{24,25}$ and therefore basically independent of temperature, or shows a significant variation with it. For such a purpose comparisons of IINS and Raman data for temperatures of 14 and $60 \mathrm{~K}$ are provided in Fig. 4. There, the approximations to $Z(\omega)$ calculated assuming linear and quadratic frequency dependences for $C(\omega)$ are shown together with the neutron spectra for the glass, the crystal Raman spectra, and that calculated from the LD frequency distribution. As can be seen, the Raman intensities $I_{\text {norm }}(\omega)$ show, in the region below $1 \mathrm{THz}$ frequency dependences $\propto \omega^{1.95}(14 \mathrm{~K})$ and $\propto \omega^{2.77}(60 \mathrm{~K})$, implying that the coupling function should depend on $\omega$ with an exponent close to unity at $14 \mathrm{~K}$ and of 1.77 at $60 \mathrm{~K}$. A quadratic dependence for $C(\omega)$ substantially underestimates the intensity for $\omega \geqslant 2 \mathrm{THz}$, as well as leads to some overestimation within the region $1.0 \mathrm{THz} \leqslant \omega \leqslant 1.5 \mathrm{THz}$ for the $60 \mathrm{~K}$ data, whereas it underestimates the intensity below $1 \mathrm{THz}$ and above $2 \mathrm{THz}$ for the spectrum at $14 \mathrm{~K}$.

Such a disagreement arises from the rather different behavior with temperature of the Raman and neutron data as shown in Figs. 4(b) and 4(c). Contrary to assertions made in Ref. 8, relatively large frequency shifts are observed in both Raman and neutron data at temperatures well below $T_{g}$. As can be seen from the graphs, the strong shifts seen in the neutron distributions in passing from low $(14 \mathrm{~K})$ to moderate $(40 \mathrm{~K})$ temperatures are not followed to the same extent by the Raman functions. The implications of such a finding can be better expressed if for a given set of temperatures the $C(\omega)$ coupling function is estimated from the Raman intensities and the neutron $Z(\omega)$ as

$$
C(\omega)=I_{\text {norm }}(Q, \omega) \omega / Z(\omega),
$$

a result which is shown in Fig. 5. Such a strong dependence of the coupling function upon temperature can be explained from consideration of the relatively large frequency shifts derived from the neutron data and shown in Fig. 5(b) in terms of the temperature dependence of the parameters characterizing the $Z(\omega)$ distributions. ${ }^{27}$ The origin of such shifts can be explained in part by the noticeable multiphonon contribution which becomes more pronounced as the temperature is raised. To see this, a multiphonon expansion, ${ }^{28}$ using data at the lowest temperature $(5 \mathrm{~K})$ as a representation of the one-phonon term was employed. Although such a simple approximation can only semiquantitatively account for the variations in the shape of the measured $Z(\omega)$, it reproduces the observed trends (a shift towards higher frequency of the maxima and noticeable broadening of the distributions as the temperature is raised). On the contrary, the same approximation fails when applied to the Raman spectra since it predicts trends at odds with what is observed. Such a result seems to 

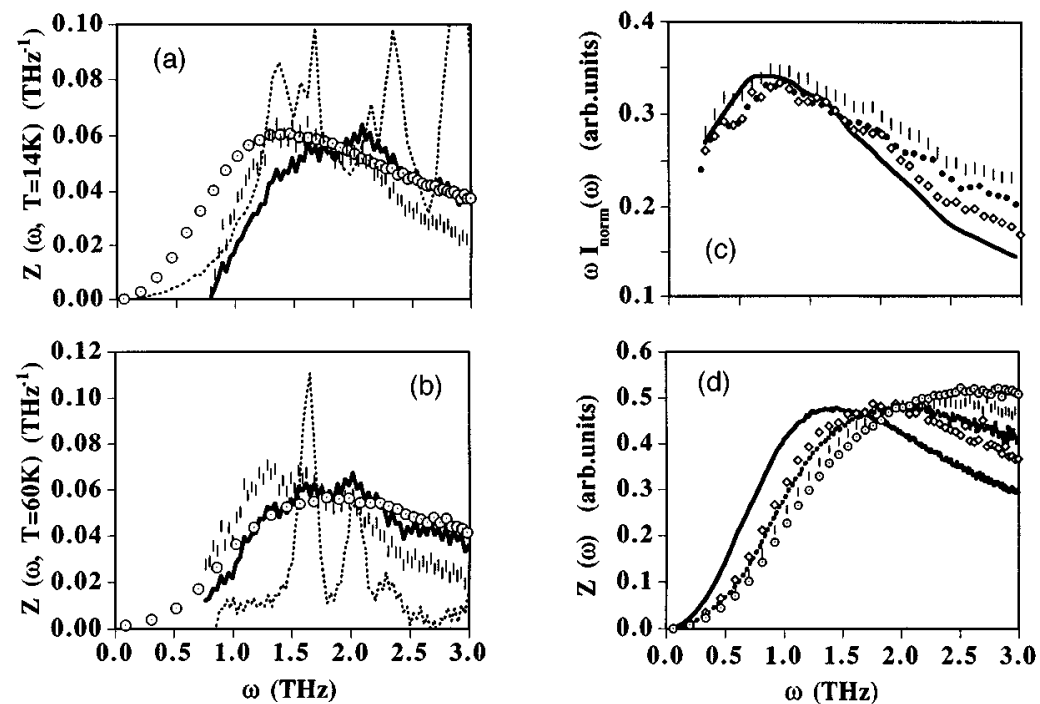

FIG. 4. A comparison between the frequency distributions derived from neutron scattering for the glass (open circles with a dot), those from Raman data assuming a quadratic dependence for $C(\omega)$ (vertical bars), a linear dependence (solid line), and the crystal spectrum (dotted line). (a) Spectra for $T=14 \mathrm{~K}$. The dotted line shows the crystal spectra as given by the low-frequency region of the distribution calculated by lattice dynamics; (b) spectra for $T=60 \mathrm{~K}$. The dotted line shows the crystal spectrum for the same temperature; (c) comparison between estimates for the $Z(\omega)$ frequency distributions derived from Raman spectra assuming a quadratic coupling. The spectra has been scaled to a common value at $1.2 \mathrm{THz}$ to facilitate the comparison of line shapes. The solid line shows the results for $T=10 \mathrm{~K}$, lozenges for $T=60 \mathrm{~K}$, filled circles for $T=120 \mathrm{~K}$, and vertical bars for $T=175 \mathrm{~K}$. (d) A comparison of the temperature dependence of the low-frequency distributions as derived from INS. Solid line stands for data taken at $T=14 \mathrm{~K}$, lozenges for $T=40 \mathrm{~K}$, dotted line for $T=60 \mathrm{~K}$, vertical bars for $T=100 \mathrm{~K}$, and circles with a dot for $T=175 \mathrm{~K}$. The curves have been scaled to unit area to facilitate comparison.

underline the rather different material properties sampled by both techniques. It seems worth noticing here that the neutron spectra are related to time-dependent two-point correlation functions as well as to higher-orders correlations, whereas for frequencies below those of the lowest lying opticlike excitations, the Raman intensity arises from the spectrum of bi- and higher order excitations involving three and four-particle correlations. Under such circumstances it is not surprising to find a discrepancy in the temperature dependence of both spectra like the one here reported.

\section{Thermodynamics}

The thermodynamic functions for the crystal are evaluated from the $Z(\omega)$ derived from calculational means, and a comparison between the $C_{v}^{\mathrm{LD}}$ constant volume heat capacity and that measured (at constant pressure $C_{p}$ ) (Ref. 29) is provided in Fig. 6(a). It shows that the present harmonic LD model can reasonably describe the thermodynamics of the crystal up to at least $T=150 \mathrm{~K}$. An estimate of the importance of the anharmonic contributions to $C_{p}$ is given by the difference $C_{p}-C_{v}^{\mathrm{LD}}$, which includes contributions arising from phonon lifetime effects as well as those associated with volume expansion. Below $150 \mathrm{~K}$ the anharmonic contributions to $C_{p}$ never exceed some $4 \mathrm{~J} \mathrm{~mol}^{-1} \mathrm{~K}^{-1}$, which at $T=100 \mathrm{~K}$ represents some six percent of the total heat capacity. Far more substantial deviations from harmonic behavior start to be noticeable above $160 \mathrm{~K}$ and become more pronounced above $250 \mathrm{~K}$.

The anharmonic contributions can be estimated from the procedure suggested by Hui and Allen ${ }^{30}$ which gives the constant pressure heat capacity, up to a first order, as ${ }^{30}$

$$
C_{p}(T)=13 R \int_{0}^{\infty} d \omega Z(\omega, T) \frac{x^{2}}{\sinh ^{2}(x)}\left[1-\frac{\partial \ln \omega}{\partial \ln T}\right]_{p}
$$

with $x=\hbar \omega / 2 k_{B} T$, temperature-dependent densities of states $Z(\omega, T)$, and a term giving the variation with temperature of the phonon frequencies. Since such contributions are known to be important only at relatively high temperatures, taking a high-temperature limit seems justified, ${ }^{30}$ and this gives a correction of the harmonic result as

$$
\Delta C \cong-C_{v}(T)\left(\frac{\partial\langle\ln \omega\rangle}{\partial \ln T}\right)_{p}
$$

where the isobaric derivatives of $\langle\ln \omega\rangle \equiv \int_{0}^{\infty} d \omega I_{\text {norm }}^{c}(\omega) \omega$ were estimated by integration of the crystal Raman spectra $I_{\text {norm }}^{c}$. Such a correction becomes specially relevant above some $200 \mathrm{~K}$ as shown in Fig. 6(a) and accounts for about one-third of the difference between $C_{p}(T)$ and its harmonic value at temperatures close to melting. The small but systematic underestimation of $C_{p}(T)$ should mostly arise from thermal expansion effects which contribute with a term $T \gamma_{G} \alpha$, where $\gamma_{G}$ is the Grüneisen constant and $\alpha$ the linear expansion coefficient, which have not been evaluated due to the lack of thermal expansion data.

As far as the glass is concerned, including the point corresponding to the supercooled liquid, the heat capacity was evaluated following Eq. (2), using the frequency distribution derived from the Raman spectra. To proceed, recourse to a 

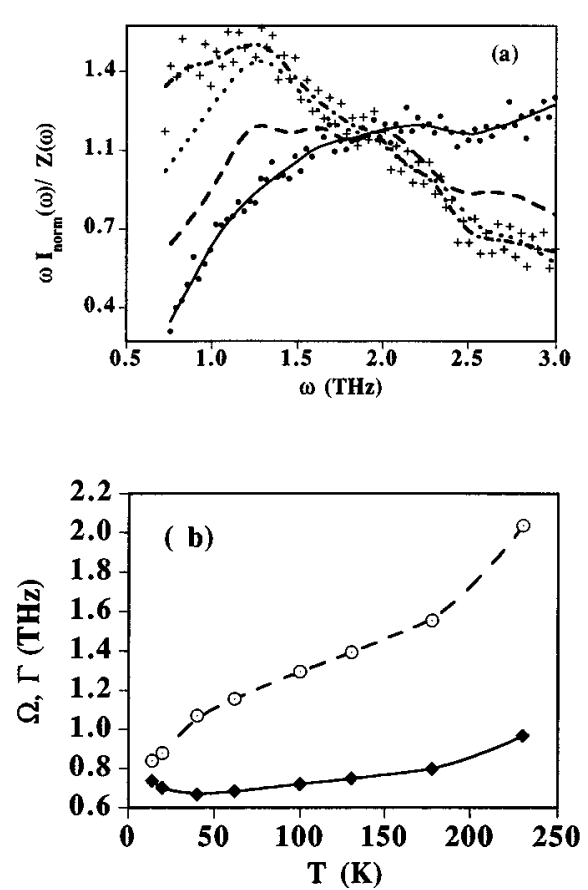

FIG. 5. (a) Estimates from the light-to-vibrations coupling function $C(\omega)$ as derived from Eq. (1) using the normalized Raman intensities and the $Z(\omega)$ from IINS as input. The lines shown correspond to approximations to data in terms of smoothing splines, and correspond to temperatures of $10 \mathrm{~K}$ (solid and filled dots), $60 \mathrm{~K}$ (dashes), $120 \mathrm{~K}$ (dots), and $170 \mathrm{~K}$ (dash-dots and crosses). The original (unsmoothed) data are shown for $10 \mathrm{~K}$ and $170 \mathrm{~K}$ only. (b) Temperature dependence of the parameters characterizing the neutron $Z(\omega)$ distributions. $\Omega$ (circles with a dot) shows the centroid of the log-normal distribution and $\Gamma$ its width (Ref. 27).

scaling constant is needed to relate the frequency integrals over the distributions to thermodynamic properties. ${ }^{31}$ Such a scale factor was found by comparison of the harmonic heat capacity evaluated from the Raman spectra corresponding to $90 \mathrm{~K}$ with the value of the experimental $C_{p}$ for such a temperature (the lowest for which thermal and Raman data are compatible), yielding a value of 5.8. Notice that such scaling is then used to find estimates of the thermodynamic functions for a temperature range between 8 and $190 \mathrm{~K}$. The result of such an exercise compares well with the experimental data, including the point within the supercooled liquid state, as can be seen from Fig. 6(b). Although such a result may appear surprising if account is made of the complicated behavior of $C(\omega)$ commented above, it is a rather direct consequence of the substantial contribution of the high-frequency spectrum arising from internal or mixed molecular modes to any property defined in terms on integrals over the frequency distribution.

Finally, Fig. 6(c) compares the average $\left\langle u^{2}\right\rangle$ atomic mean-square displacements (m.s.d.) as calculated for the glass and crystal from the relevant frequency distributions and data taken from a neutron scattering study. ${ }^{32}$ As expected, the glass data lay above the harmonic curve within the whole range of temperatures, and the marked change in slope about $180 \mathrm{~K}$ unveils the presence of the glass transition at $T_{g} \approx 185 \mathrm{~K}$. A comparison between the previous estimates for $\left\langle u^{2}\right\rangle$ from neutron scattering and the present set shows an extremely good agreement for temperatures above $100 \mathrm{~K}$, whereas the neutron data below such temperature are somewhat below the present ones for both glass and crystal. Since the present LD model adequately reproduces both the crystal structure and thermodynamics within such range of temperatures, it seems clear that the previous neutron data somewhat underestimate the value of the atomic displacement below $100 \mathrm{~K}$.

\section{CONCLUSIONS}

The present study provides a microscopic model which can be used to explore the most relevant details concerning the structure and dynamics of the disordered phases of solid glycerol at least on a semiquantitative basis.

From results given above, it should become clear that the understanding of the thermodynamics of glass and crystal as well as other properties defined as integrals over the frequency distribution at temperatures close to $T_{g}$ requires the

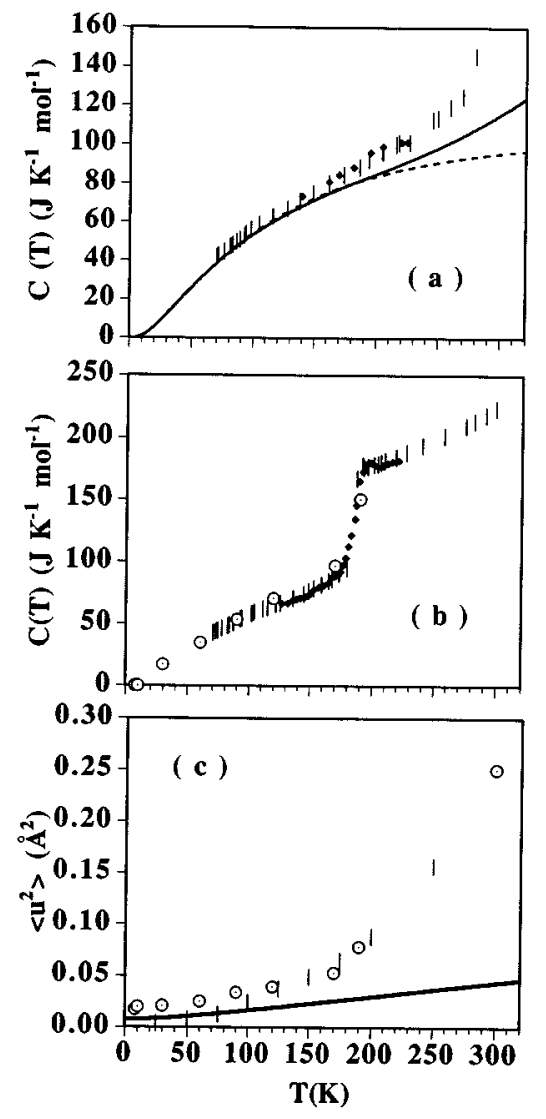

FIG. 6. (a) A comparison between the experimental heat capacity (vertical bars from Ref. 29 and lozenges from Ref. 2), the calculated function in the harmonic approximation (dashed line), and that including the anharmonic contribution given by Eq. (2) (solid line). (b) Experimental heat capacity data for the glass and liquid (vertical bars from Ref. 29 and lozenges from Ref. 2) and that calculated from the approximations to $Z(\omega)$ as calculated from the Raman frequency distributions (circles with a dot). (c) Average atomic mean square displacements as calculated for glass and liquid samples by integration of the Raman frequency distributions (open circles with a dot), those for the harmonic crystal (solid line), and those taken from Fig. 3(a) of Ref. 32 (see text) (vertical bars). 
explicit consideration of at least the lowest seven molecular degrees of freedom. Any rigid-molecule model could only describe the dynamics of both phases below some $70 \mathrm{~K}$.

The present results help to explain the origin of the substantial deviations from simple-liquid behavior recently found for frequencies above some $0.3 \mathrm{THz}$ in studies carried at temperatures close but above $T_{g},{ }^{23}$ since the strong couplings between density fluctuations and internal molecular large-amplitude motions extend to rather small frequencies. In fact, the onset of microscopic behavior found in the crystal $Z(\omega)$ for frequencies above $0.6 \mathrm{THz}$ sets an absolute limit for the applicability of the MCT predictions ${ }^{8}$ regarding the $\omega^{a}$ power law for the susceptibility.

On the other hand, even if our data only regard the glass phase, the remarkable dependence of $C(\omega)$ with temperature serves to explain the disagreement between light-scattering (Brillouin and Raman) and neutron results found in tests of some theoretical predictions regarding the temperature dependence of the susceptibility. ${ }^{8}$

"Present address: Institut Laue Langevin, BP 156X, F-38042 Grenoble Cedex 9, France.

${ }^{1}$ R. L. Cook, H. E. King, C. A. Herbst, and D. R. Herschbach, J. Chem. Phys. 100, 5178 (1994).

${ }^{2}$ See, for instance, C. Allain and P. Lallemand, J. Phys. (Paris) 40, 693 (1979).

${ }^{3}$ N. O. Birge, Phys. Rev. B 34, 1631 (1986); M. Massalska-Arodź, ibid. 43, 13676 (1991).

${ }^{4}$ W. Tandy Grubbs and R. A. McPhail, J. Chem. Phys. 100, 2561 (1994); See also R. Vacher and J. Pelous, J. Chim. Phys. 82, 311 (1985).

${ }^{5}$ S. Kojima, Phys. Rev. B 47, 2924 (1993).

${ }^{6}$ M. Grimsditch and N. Rivier, Appl. Phys. Lett. 58, 2345 (1991).

${ }^{7}$ N. Menon, K. P. O’Brien, P. K. Dixon, L. Wu, and S. R. Nagel, J. Non-Cryst. Solids 141, 61 (1992); N. Menon and S. R. Nagel, Phys. Rev. Lett. 74, 1230 (1995).

${ }^{8}$ J. Wuttke, J. Hernandez, G. Li, G. Goddens, H. Z. Cummins, F. Fujara, W. Petry, and H. Sillescu, Phys. Rev. Lett. 72, 3052 (1994). The frequency dependence found for the susceptibility above the minimum follows a $\omega^{a}$ behavior with exponent values which are strongly dependent on the source of data ( $a=0.32$ for light scattering and $a=0.36$ for neutrons), as well as on the constraints introduced for the fit, which are a central result from MCT predictions. Substantially better fits are found if no constraints are used but the parameter values increase up to $a=0.56$.

${ }^{9}$ H. B. Hass and J. A. Patterson, Ind. Eng. Chem. 33, 615 (1941).

${ }^{10}$ A more complete account of the neutron measurements including experimental results concerning the collective dynamics as recently explored using the MARI spectrometer at ISIS will be given elsewhere.

${ }^{11}$ H. van Koningsveld, Rec. Trav. Chim. 87, 243 (1968).

${ }^{12}$ O. Bastiansen, Acta Chem. Scand. 3, 415 (1949).

${ }^{13}$ L. J. Root and F. H. Stillinger, J. Chem. Phys. 90, 1200 (1989).

${ }^{14}$ D. E. Williams, J. Chem. Phys. 47, 4680 (1967); S. R. Cox, D. E. Williams, and L. Hsu, Acta Crystallogr. A37, 293 (1981); A. Criado and A. Muñoz, ibid. B49, 726 (1993).

${ }^{15}$ E. R. Lippincott and R. Schroeder, J. Chem. Phys. 23, 1099 (1955).

${ }^{16}$ J. L. Derissen and P. H. Smit, Acta Crystallogr. A34, 842 (1978).
Finally, a word of caution against oversimple comparisons seems in order in the light of the present data and those regarding a van der Waals glass previously considered. ${ }^{19}$ In both cases strong hybridization effects between internal and external modes were noted and, as a matter of fact, substantial couplings down to $0.65 \mathrm{THz}$ were found for OTP. ${ }^{19} \mathrm{~A}$ Boson peak is also present in the latter material at some 0.4 $\mathrm{THz},{ }^{22}$ at a lower frequency than in glycerol. In consequence the lesser strength in OTP of the microscopic dynamics cannot be the only reason explaining the better agreement with MCT predictions.

\section{ACKNOWLEDGMENTS}

This work has been supported in part by DGICYT Grant No. PB92- 0114-C04. The authors wish to thank Dr. M. Garcia-Hernandez and Dr. F. J. Mompeán for invaluable help given during the neutron measurements at the Siloé reactor.

${ }^{17}$ W. R. Busing, Acta Crystallogr. A28, S252 (1972).

${ }^{18}$ G. Taddei, H. Bonadeo, M.P. Marzzochi, and S. Califano, J. Chem. Phys. 58, 966 (1973).

${ }^{19}$ A. Criado, F.J. Bermejo, A. de Andrés, and J.L. Martinez, Mol. Phys. 82, 764 (1994).

${ }^{20}$ See AIP document no. PAPS PRMDO-53-5259-3 for 3 pages of tabular material containing the eigenvector coefficients. Order by PAPS number and journal reference from American Institute of Physics, Physics Auxiliary Publication Service, Carolyn Gehlbach, 500 Sunnyside Boulevard, Woodbury, New York, 11797. Fax: 516-576-2223, e-mail: janis@aip.org. The price is $\$ 1.50$ for each microfiche (98 pages) or $\$ 5.00$ for photocopies of up to 30 pages, and $\$ 0.15$ for each additional page over 30 pages. Airmail additional. Make checks payable to the American Institute of Physics.

${ }^{21}$ F. J. Bermejo, M. Garcia-Hernandez, B. Fåk, J. Alonso, J. L. Martinez, and T. Mason, Europhys. Lett. 24, 545 (1993).

${ }^{22}$ F. J. Bermejo, A. Criado, and J. L. Martinez, Phys. Lett. A 195, 236 (1994).

${ }^{23}$ E. Rössler, A. P. Sokolov, A. Kisliuk, and D. Quitmann, Phys. Rev. B 49, 14967 (1994).

${ }^{24}$ T. Achibat, A. Boukenter, E. Duval, B. Frick, N. Garcia, and J. Serrughetti, Physica A 201, 257 (1993).

${ }^{25}$ E. Duval, N. Garcia, A. Boukenter, and J. Serrughetti, J. Chem. Phys. 99, 2040 (1993); T. Achibat, A. Boukenter, and E. Duval, ibid. 99, 2046 (1993).

${ }^{26}$ J. Jäckle, in Amorphous Solids: Low Temperature Properties, edited by W. A. Phillips (Springer-Verlag, Berlin, 1981), p. 181.

${ }^{27} \mathrm{~A}$ normalized log-normal distribution given as $A \exp [-\log (w)-\Omega]^{2} / 2 \Gamma^{2} / \Gamma \omega \sqrt{2 \pi}$ given in terms of three parameters specifying a scale factor $(A)$, its centroid $(\Omega)$, and width $(\Gamma)$, reproduces rather satisfactorily the shape of $Z(\omega)$. Such a fact was commented on by V. K. Malinovski, V. N. Novikov, P. P. Parshin, A. P. Sokolov, and M. G. Zemlyanov, Europhys. Lett. 11, 43 (1990).

${ }^{28}$ The usual multiphonon expansion following the incoherent approximation was adopted. A brief account of the approximation is given by S. W. Lovesey, Theory of Neutron Scattering from Condensed Matter (Oxford Science, Oxford, 1984), p. 160.

${ }^{29}$ G. E. Gibson and W. F. Giauque, J. Am. Chem. Soc. 45, 93 
(1923). Some more recent measurements regarding a narrower range of temperatures are given by $\mathrm{M}$. Rajeswari and $\mathrm{A}$. K. Raychaudhuri, Phys. Rev. B 47, 3036 (1993); and low temperature $(\approx 1.5-4 \mathrm{~K})$ data have been taken from R. S. Craig, C. W. Massena, and R. M. Mallya, J. Appl. Phys. 36, 108 (1965).
${ }^{30}$ J. C. K. Hui and P. B. Allen, J. Phys. C 8, 2923 (1975).

${ }^{31}$ A. P. Sokolov, A. Kisliuk, M. Soltwisch, and D. Quitmann, Phys. Rev. Lett. 69, 1540 (1992).

${ }^{32}$ F. Fujara, W. Petry, R. M. Diehl, W. Schnauss, and H. Sillescu, Europhys. Lett. 14, 563 (1991). 